\title{
A Large Gystie Degenerating Broad ligament Leiomyoma Masquerading as Ovarian Malignancy!
}

Renuka Malik, Garima Agarwal

Department of Obstetrics and Gynecology, PGIMER, Dr. Ram Manohar Lohia hospital, New Delhi 110001 , India.

Abstract:

We report an unusual case of a large, broad ligament uterine leiomyoma with cystic degenerations, masquerading as a malignant ovarian tumor on ultrasonography and CT with the diagnostic difficulties posed. A 40-year-old married female presented with complaints of abdominal distention and a palpable abdominopelvic mass occupying whole of lower abdomen. Ultrasonography and CT revealed a large solid mass with cystic areas extending into both uterine adnexa. The patient underwent a laparotomy. Gross examination revealed normal ovaries and a pedunculated mass with prominent cystic honeycomb degenerations, originating from the uterus. The tumor was excised through the peduncle and pathologic evaluation revealed a uterine leiomyoma with cystic degeneration. In conclusion, a large pedunculated leiomyoma with cystic degenerations can mimic a malignant ovarian neoplasm on imaging studies. Therefore, uterine leiomyoma with cystic degenerations should be considered during the differential diagnosis of large adnexal masses.

Key words: Abdomen, Broad Ligament, Leiomyoma, Ovarian Neoplasms, Uterine Malignancy.

\section{Introduction}

Leiomyoma of the uterus is the most common benign tumor of the female pelvis in the reproductive age group, which arises from uterine smooth muscle, varying in size from microscopic to giant, although the latter being exceedingly rare. They may be asymptomatic in more than $50 \%$ of patients or present with heavy menstrual bleeding, infertility or pressure effects if large or with pain if undergoing torsion as in case of pedunculated fibroid, expulsion if submucosal fibroid polyp or rapid growth as in sarcomatous change. They have typical easily recognizable appearance on imaging, however the atypical appearances that follow degenerative change can cause diagnostic dilemma. Confusion in imaging studies may arise when fibroids present in unusual locations or undergo degenerations, which must be kept in mind. Here, we present a case of a woman with giant uterine myoma that had undergone extensive cystic degenerative changes, camouflaging an ovarian malignancy.

Corresponding Author: Dr. Renuka Malik

Email: renucam@yahoo.co.in

Received: September 24, 2015 | Accepted: November 7, 2015 | Published Online: November 15, 2015 This is an Open Access article distributed under the terms of the Creative Commons Attribution License (creativecommons.org/licenses/by/3.0)

Conflict of interest: None declared | Source of funding: Nil | DOl: http://dx.doi.org/10.17659/01.2015.0125 


\section{Case Report}

A 40 year old para 3 live 3 presented to the outpatient department of Dr. Ram Manohar Lohia Hospital with a history of gradually progressive abdominal distension for 12 months with insidious onset of diffused, dull aching and intermittent abdominal pain for 3 months. She had a weight gain of approximately $8 \mathrm{kgs}$ with a feeling of heaviness and hardness in the lower abdomen. History of nausea, vomiting, bloating, dyspepsia, single episode of hematemesis, feeling of early satiety and constipation was present. No history of fever, anorexia or any chronic medical/surgical illness was reported. She had regular and cyclical menstrual pattern.

Examination revealed a middle aged lady of average build and nutrition. Her vital signs were within normal limits. Systemic examination was normal. Entire lower abdomen was uniformly distended by a $25 \times 20 \mathrm{~cm}$ mass with smooth surface, regular well defined margins, side to side mobility, non-tender mass cystic in consistency arising from, pelvis reaching $4 \mathrm{~cm}$ above umbilicus. Shifting dullness was absent. Per speculum examination revealed a healthy cervix and vagina. Per vaginum examination revealed that cervix was firm and downwards but deviated to the left side; uterus was normal-sized, anteverted and mobile. The same mass was felt through all fornices with cervical movements not being transmitted to mass. Per rectal examination was normal.

Complete blood count, serum electrolyte levels, tests of liver and renal function, pap smear and endometrial sampling were normal. Ultrasonography and CECT revealed a huge $22 \times 20 \mathrm{~cm}$ abdominopelvic cystic lesion with multiple internal septations and solid components likely arising from right adnexa suggestive of primary epithelial ovarian malignancy (serous cystadenocarcinoma) without ascites [Fig.1]. However, the tumor markers were within normal limits. Upper gastrointestinal endoscopy was normal.

With a probable diagnosis of ovarian tumor, the patient was taken up for staging laparotomy. An abdominal midline xiphopubic vertical incision was made. Peritoneal washings were taken from paracolic gutters, pouch of Douglas and sent for cytology. It was seen that uterus and bilateral ovaries were normal. Enclosed between the leaves of broad ligament a large $25 \times 20 \mathrm{~cm}$, smooth, cystic mass was seen attached by a small $3 \mathrm{~cm}$ stalk to the right lateral wall of uterus between right round ligament and right uterine artery and pushing the right ovary posteriorly. Enucleation of the mass was done after opening the right broad ligament and dividing the round ligament. There was a $3 \times 3 \mathrm{~cm}$ peduncle attached to the right lateral uterine wall above uterine artery, which was cut and transfixed. The normal uterus and ovary were conserved after this myomectomy.

Gross histopathology specimen showed $25 \times 20 \mathrm{~cm}$ smooth, multiloculated cystic mass with intact capsule weighing $2.2 \mathrm{~kg}$ [Fig.2]. 2-litre straw colored fluid drained from cut section and sent along with cyst wall for histopathology. It was honeycomb with multiple thin septations filled with gelatinous and mucoid material with hyaline degeneration and

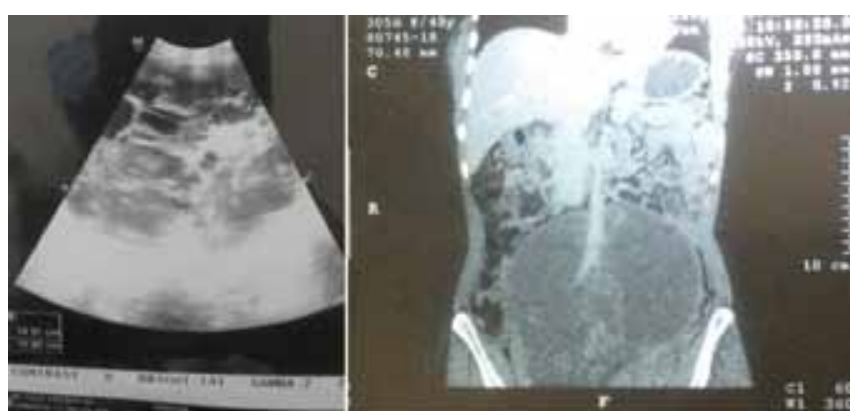

Fig.1: Ultrasound and CT reported as ovarian tumor. 
calcification. Microscopy showed benign spindle cell tumor of the stroma tissue origin with marked cystic degeneration. Final impression was leiomyoma with marked hyaline and cystic degeneration. Postoperative recovery of patient was uneventful.

\section{Discussion}

Leiomyoma of the uterus is the most common tumor of female pelvis with a prevalence of 20\%-30\%. Extra-uterine location of fibroids are rare. Leiomyoma of uterus arises from uterine smooth muscle. The size, number and location determine their clinical presentation. It can be single or multiple, size can vary from small to big. They can be submucosal, intramural or subserosal which may be pedunculated and simulate adnexal mass. In $50 \%$ of cases there are no symptoms [1].

The extra-uterine location of leiomyoma can be in broad ligament, ovary, urinary bladder, urethra, vulva, vagina or anywhere there is smooth muscle. Other variants in growth patterns can be diffuse peritoneal leiomyomatosis, intravenous leiomyomatosis, parasitic leiomyoma or benign metastasizing leiomyomatosis, where they can be found even in lung due to hematogenous dissemination and retroperitoneal leiomyomas [2]. In the broad ligaments the fibroid can be of two types i) true broad ligament fibroid which can arise from smooth muscle of round ligament, tubo-ovarian ligament or smooth muscle of uterine artery or ovarian vessels; ii) false broad ligament fibroid which arises from lateral wall of uterus or cervix. Leiomyoma occasionally occurs with unusual growth pattern or in unusual locations that makes their identification more challenging, both clinically and radiologically.

Most common secondary changes are degeneration, infection, hemorrhage, necrosis and rarely sarcomatous change. Degeneration like cystic,
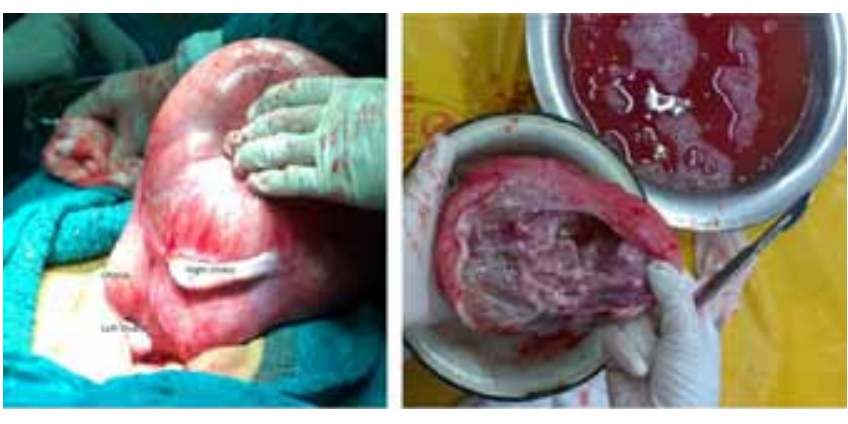

Fig.2: Gross specimen and cut section of the tumor.

hyaline, myxoid or red degeneration occur when fibroids outgrow their blood supply. Calcification follows necrosis. Out of these, hyaline degeneration is common occurring in about $60 \%$ of cases whereas cystic degeneration is rare in about $4 \%$. These degenerations pose a diagnostic challenge in imaging studies. The typical appearance of leiomyoma is easily recognized on ultrasound but degenerations, as in our case may cause confusion in diagnosis. CT is not the primary modality in the diagnosis of fibroid [3]. MRI is not cost effective for diagnosis of fibroids, used only in doubtful cases and in unusual locations. Imaging studies too have their pit falls as seen in our case.

Our case was a false broad ligament fibroid which arose out from uterus and grew within the folds of broad ligament with marked cystic degeneration. Degenerating fibroid can mimic ovarian tumor, endometrioma and abscess on ultrasound. Ultrasound is the diagnostic modality for fibroids. They present with varying echogenicity, depending upon their relative fibrous tissue to muscle ratio. Typically a fibroid on ultrasound appears solid in echogenicity with hypogenic shadowing. Degenerations give heterogeneous appearance. However, appearance of normal ovary should have been seen in our case which was missed on both ultrasound and in $\mathrm{CT}$ by radiologist. 


\section{Conclusion}

Although fibroids typically have a characteristic ultrasound appearance, degenerating fibroids can have variable patterns and pose diagnostic challenges. Ours is an unusual case of a pedunculated leiomyoma with extensive cystic degeneration presenting with symptoms of gastro intestinal pathology, masquerading as a cystic epithelial ovarian tumor. We are reporting this case on account of its rarity and diagnostic difficulties.

\section{References}

1. Gupta S, Jose J, Manyonda I. Clinical presentation of fibroids. Best Pract Res Clin Obstet Gynaecol. 2008;22(4):615-626.

2. Fasih N, Prasad Shanbhoque AK, Macdonald $D B$, Fraser-Hill MA, Papadatos D, Kielar AZ, et al. Leiomyomas beyond the uterus: unusual location, rare manifestations. RSNA Radiographics. 2008;28(7):1931-1948.

3. Wilde S, Scott-Barrett S. Radiological appearances of uterine fibroids. Indian J Radiol Imaging. 2009;19:222-231. 